\title{
Éditorial
}

\section{L'exploitation agricole : un concept à revisiter du nord aux suds}

Jean-Pascal Pichot

Chargé de mission,

Centre de coopération internationale en recherche agronomique pour le développement (Cirad) <portapich@aol.com> es articles présentés dans ce numéro thématique des Cahiers Agricultures illustrent une diversité de points de vue sur une structure centrale, une institution, du monde rural - l'exploitation agricole - dont on pourrait s'étonner qu'elle suscite encore l'intérêt des chercheurs tant les agronomes et les économistes l'ont investie au cours des dix-neuvième et vingtième siècles dans les pays du Nord.

Ce concept d'exploitation agricole issu des recherches sur les sociétés rurales européennes a en effet été beaucoup utilisé comme grille d'analyse de la diversité des structures de production (typologies, trajectoires) et comme support ou cible des politiques publiques nationales ou européennes concernant l'agriculture (sensu lato).

Combinaison durable de forces de travail, de savoir-faire, de droits d'accès (sécurisés par le droit) à des ressources naturelles ou construites, de bâtiments et de cheptels, l'exploitation agricole "européenne " moderne était un patrimoine transmis d'une génération à l'autre, elle s'inscrivait dans l'histoire de territoires ruraux en évolution lente. N'est-elle pas aujourd'hui en mutation rapide, voire en voie de disparition?

Après 40 ans de politique agricole de soutien aux productions végétales et animales, le nombre des exploitations agricoles en France comme en Europe diminue de façon inquiétante, faute d'exploitants malgré les aides à l'installation des jeunes. Le risque de voir se distendre, ici ou là, les liens sociaux qui fondent les territoires et disparaitre les services publics indispensables aux ménages ruraux, va donc croissant.

Parfois qualifiée de familiale lorsqu'elle assurait l'emploi et bénéficiait de la force de travail de ménages ruraux composés de la famille du chef d'exploitation (épouse, ascendants et descendants), l'exploitation agricole a donné lieu à des représentations et discours syndicaux mais aussi politiques destinés à la mythifier alors même que les familles éclataient, diversifiaient leurs activités et sources de revenus, substituaient les équipements à la main-d'oeuvre et nouaient des alliances pour externaliser diverses fonctions/emplois. Cette mutation est particulièrement nette pour les grandes cultures où certains chantiers de travaux - la stabilisation postrécolte des produits, leur transformation, leur commercialisation - peuvent être " confiés " à des coopératives, des Sica (société d'intérêt collectif agricole), ou des entreprises, pour optimiser économiquement la production de produits standardisés. Ce modèle d'exploitation pilotée par un manager est-il porteur d'avenir dans un contexte de libéralisation des échanges? Est-il résilient, durable, adapté aux attentes d'une société urbanisée?

L'exploitation "familiale" fondée sur le travail et la solidarité familiale peut-elle aujourd'hui se ressourcer en Europe au travers de diverses fonctions d'intérêt général :

- diversification et qualification de la production, transformation et commercialisation de produits destinés à des marchés de niches (produits bio, ventes à la ferme, réseaux de commercialisation alternatifs en milieu urbain) ou bénéficiant d'attributs spécifiques liés à des savoir-faire ou des territoires?

- services environnementaux, sociaux ou culturels encouragés, il fut un temps, par une politique d'appui aux fonctions non directement productives de biens marchands agricoles (contrat territorial d'exploitation (CTE) en France).

La diversification des fonctions de l'agriculture en réponse à des demandes 
sociétales nouvelles pourra-t-elle lui redonner du sens et freiner la déprise? En dehors de son berceau originel européen, le concept d'exploitation agricole, véhiculé non seulement par les experts des bailleurs de fonds internationaux, des agences de coopération ou des organisations non gouvernementales (ONG), mais aussi par les chercheurs et enseignants, a été utilisé sans beaucoup de précautions pour identifier des projets de développement rural dans des sociétés où les modalités d'accès aux ressources, l'organisation du travail, voire les finalités de l'activité agricole et les formes de capitalisation étaient, et restent, fort éloignées du modèle standard européen. Cet impérialisme idéologique a fondé depuis plusieurs décennies les politiques agricoles de nombreux pays en développement ou de pays émergents, malgré des alertes répétées de quelques chercheurs en sciences sociales mieux au fait des réalités sociales et culturelles de ces pays. Les succès modestes de ces politiques en termes de "développement " rural ou de sortie de la production agricole du secteur informel (en dehors des filières d'exportation, bien sûr) ne doivent-ils pas nous interpeller vigoureusement?

N'est-il pas temps d'accepter, au moins comme hypothèse de travail, la possibilité de voir coexister durablement au sein des pays et des régions des modèles contrastés d'organisation du monde rural et de l'activité agricole?

Cette réalité n'est aujourd'hui pas vue comme durable selon les critères de performances des techniciens ou des économistes. Ne faudra-t-il pas désormais élargir l'analyse en prenant en compte des critères sociaux, environnementaux, politiques?

Les agronomes ont formalisé depuis plusieurs décennies un cadre d'analyse de l'exploitation agricole fondé sur la production agricole de produits végétaux ou animaux. Ce cadre d'analyse semble parfaitement adapté aux agricultures pilotées par les échanges marchands de produits standardisés et par les subventions à la production accordées par certains États au titre de la solidarité nationale ou du développement territorial. Il convient bien, aussi, aux représentants professionnels qui revendiquent, de façon presque incantatoire, la production agricole comme finalité première. Il convient enfin aussi aux services d'appuis, aux banques agricoles et aux concepteurs des politiques de soutien au secteur agricole, qui identifient ainsi leurs " clients ".
Des lézardes se font cependant jour peu à peu depuis que les finalités de l'agriculture s'élargissent à la production d'aménités diverses concernant l'environnement, l'accueil des populations urbaines en zones rurales et périurbaines, la qualification identitaire des produits, leur transformation à la ferme, leur distribution dans des réseaux associatifs...

Des difficultés se font jour aussi quand on applique sans nuances ce cadre d'analyse à des situations agricoles ne partageant pas la même histoire et où les sociétés rurales n'ont pas tout sacrifié à la spécialisation des espaces et des activités ou aux échanges marchands.

Sans chercher très loin, on peut penser aux paysans des départements et collectivités territoriales françaises d'outre-mer, aux paysans brésiliens du Nordeste, aux paysans albanais, etc., qui développent des activités diverses et dont la production agricole s'inscrit dans des calendriers alimentaires domestiques, dans des échanges marchands sur des marchés de proximité et non marchands au sein des groupes familiaux.

Dans le même temps, certains économistes ont développé un cadre d'analyse et d'action dans lequel l'exploitation agricole est vue comme une entreprise, si possible distanciée par rapport au "ménage " qui la gère et en assure la pérennité

Ce cadre d'analyse, cohérent avec celui des agronomes, s'est avéré très pertinent pour développer des services d'appuis permettant d'optimiser les résultats économiques de l'exploitation en spécialisant les activités de production, en captant les subventions, et en arbitrant entre les facteurs terre, capital et main-d'œuvre, souvent en faveur du capital foncier et de l'équipement, au détriment de la maind'œuvre.

Après plusieurs décennies d'une " professionnalisation " de l'agriculture basée sur ce cadre conceptuel, des craintes se font jour, ici ou là, quant à l'usage agricole de certains milieux, voire de certaines régions, en déprise faute d'agriculteurs, d'éleveurs et de main-d'œuvre rurale. Ailleurs on s'interroge sur les risques économiques de la spécialisation (banane, canne à sucre, vigne, etc.) ou sur le coût des impacts environnementaux de certaines filières végétales et animales (pollueurs, payeurs, pollueurs-payeurs?). Dans un contexte international marqué par une tendance à la libéralisation des échanges et à la diminution des aides à la production, on peut s'interroger sur la compétitivité et la résilience, en l'absence d'aides publiques, des entreprises agricoles spécialisées dans la productiontransformation industrielle et l'exportation de produits à faible valeur ajoutée (blé, sucre, maïs, soja, lait, viande bovine, banane...).

Dans le même temps, les économistes constatent que les microstructures agricoles, dont nombre d'experts et de politiques souhaitaient et prévoyaient la rapide disparition "au profit " des moyennes et grandes exploitations, font de la résistance alors même que des politiques spécifiques, par exemple de crédit, sont destinées à les faire sortir du secteur informel et à les amener vers les échanges marchands. Dans de nombreux pays africains soumis à des plans d'ajustement structurel depuis une ou deux décennies, le secteur informel a même gagné du terrain, ce qui n'était pas vraiment prévu ni souhaité car le risque de passage de l'informel à l'illicite est évident (cultures de cannabis, etc.).

Dans bien des cas, il semble ainsi que les ressorts économiques des politiques agricoles actuelles ne suffisent pas à promouvoir des solutions durables.

Ne faudrait-il donc pas refonder le pacte social entre secteur agricole et sociétés de plus en plus urbanisées, aussi bien dans les pays postindustriels que dans les pays à dominante agricole? La question des finalités du secteur agricole et des sociétés rurales, dans des pays où la population est majoritairement (60 à $80 \%$ ) urbaine, ne semble pas différable.

La multifonctionnalité de l'agriculture estelle porteuse de sens et d'avenir pour les sociétés rurales et pour certaines exploitations agricoles? La chimie verte et la production de biocarburants pour d'autres? Des sociétés rurales duales existent, par exemple au Brésil ou dans les départements français d'outre-mer où des filières de production et des exploitations orientées vers le marché coexistent avec des exploitations de petite taille privilégiant d'autres finalités et une pluralité d'activités. Pourquoi devrions-nous ignorer ces dernières ou essayer de les faire disparaitre? Ne faut-il pas au contraire s'attacher à mieux comprendre leurs finalités et adapter nos cadres d'analyse, en mobilisant de nouvelles disciplines dans des approches pluridisciplinaires?

Au plan des finalités, la reproduction du groupe domestique (alimentation, habitation, santé) et ses relations/obligations 
d'entraide ou de solidarité avec les autres groupes du lignage, du groupe familial élargi, ou de la communauté locale, justifient mieux la diversité des systèmes d'activité, l'entrelacement des échanges marchands et non marchands que la recherche du profit économique.

Au plan des méthodes, vouloir isoler les unités familiales et en faire des typologies n'est peut-être pas pertinent, si ce qui fait sens tient d'abord aux relations entre ces unités. L'analyse des chantiers de travaux collectifs, des calendriers alimentaires et de trésorerie, des échanges temporaires de terres ou de matériels contre du travail, l'analyse des relations de réciprocité au sein du groupe ou de la communauté, comme l'utilisation des transferts d'argent venant des migrants, sont autant de domaines encore peu prospectés. Ils s'inscrivent en effet dans un cadre où la finalité du groupe familial n'est pas la production agricole et où l'accumulation peut trouver des formes culturelles (pèlerinage, tombeau, mosquée, etc.) générant du capital social plus que du capital d'exploitation.
Un proverbe bambara dit en substance : "Le pauvre n'est pas celui qui n'a pas de chemise, c'est celui qui n'a pas d'amis. " Ce proverbe n'exprime-t-il pas le nécessaire enchâssement de l'économique dans le social? Dans les sociétés rurales où la " richesse " d'une famille est d'abord constituée de travail familial, de droits d'accès à la terre et à l'eau sécurisés socialement, d'assurances contre les aléas liées à l'appartenance à un groupe, n'est-il pas judicieux de privilégier le capital social?

Y a-t-il de bonnes raisons pour les politiques de vouloir faire sortir ces "famillessystèmes d'activités" de ces réseaux de relations qui sont de puissants moyens de lutte contre la " pauvreté "?

Au plan de la recherche et de l'intervention, ne devons-nous pas reconnaittre humblement que nous sommes confrontés à des objets complexes organisés en réseaux sociotechniques irréductibles à des approches analytiques disciplinaires?

Cette posture nouvelle n'est pas confortable, elle laisse ouvertes toutes les questions relatives aux niveaux d'agrégation pertinents, ici ou là, pour analyser, par exemple :

- les prises de décision concernant les activités agricoles au sein du groupe familial (affectation des facteurs de production internes, achats d'intrants, échanges) ou externes à celui-ci (participations à des groupes de jeunes, de femmes, à des groupements d'intérêt économique (GIE)...);

- les prises de décision concernant les migrations courtes et longues des membres de la famille;

- les arbitrages entre consommation, épargne, crédit ;

- les arbitrages entre échanges marchands et réciprocité ;

- les possibilités d'innovation technique, économique ou sociale ;

- l'entretien ou amélioration du capital social ou du capital d'exploitation.

Toutes les sociétés rurales construisent et font évoluer des règles d'accès aux ressources, des conventions relatives à l'accumulation ou à la redistribution du capital, qui ont été souvent ignorées au prétexte qu'elles relevaient du passé. N'est-il pas, enfin, temps de leur accorder toute notre attention? 